\title{
Fast multifrequency focal beam analysis for 3D seismic acquisition geometry
}

\author{
Wei Wei ${ }^{1}$, Li-yun $\mathrm{Fu}^{1}$, and Gerrit Blacquière ${ }^{2}$
}

\begin{abstract}
A method for the efficient computation of multifrequency focal beams for 3D seismic acquisition geometry analysis has been developed. By computing them for all the frequency components of seismic data, single-frequency focal beams can be extended to multifrequency focal beams. However, this straightforward method involves considerable computer time and memory requirements, especially in complex media settings. Therefore, we propose a rapid 3D multifrequency focal beam method in which only a few single-frequency focal beam computations are followed by a number of smart interpolations. The 3D wavefield extrapolation in the focal beam analysis is conducted by the combined applications of a 3D degenerate Fourier migrator and a 3D Born-Kirchhoff interpolation operator, a process that reduces the computational cost for complex media. The multifrequency focal beam analysis is applied to a 3D model from an oil field of China, demonstrating how spatial sampling differences affect seismic imaging.
\end{abstract}

\section{INTRODUCTION}

An ideal seismic acquisition survey, consisting of a dense grid ("carpet") of shots and detectors, can produce and record the ideal seismic wavefield perfectly. However, due to the constraints of exploration time and budget, a sparse geometry (such as a parallel, orthogonal, or other irregular geometry) is often adopted in practical seismic exploration applications. Sparse geometries, while reducing exploration cost, can cause uneven sampling and should be assessed for its sampling performance prior to acquisition. According to the seismic value chain (Berkhout, 2004), which represents the cyclic interaction between seismic acquisition, structural imaging, and reservoir characterization, we need to design economical acquisition geometries that potentially yield the best images in terms of spatial resolution and amplitude accuracy, as well as the best reservoir models that allow accurate estimates of recoverable reserves.

In conventional survey design, which is based on the common midpoint (CMP) analysis for a horizontally layered earth, the quality of an acquisition geometry is generally judged by such properties as CMP fold, offset distribution, and azimuth distribution; see Cordsen et al. (2000) and Vermeer (2002) for details. For a complex subsurface structure, a common reflection point (CRP) analysis can be used to evaluate the number and angle of rays passing through a target bin by ray tracing in a velocity model. As for CMP analysis, CRP analysis also produces the attributes of fold, offset, and azimuth in target bins (Slawson et al., 1994; Muerdter and Ratcliff, 2001; Chang et al., 2002). Furthermore, now that prestack migration is in the mainstream of industry techniques for seismic data processing, 3D seismic survey analysis is often based on the performance of prestack migration, rather than traditional stacked seismic data. A survey analysis based on prestack migration is able to provide a direct estimate of the final image quality at a particular target area, rather than the indirect attributes computed at the surface by a traditional survey analysis.

Focal beam analysis (Berkhout et al., 2001; Volker et al., 2001; Volker, 2002) is a method that relates prestack migration theory (Berkhout, 1987) directly to the assessment of seismic acquisition geometries. As opposed to spatial resolution analysis (Vermeer, 1999; Gibson and Tzimeas, 2002; Tzimeas, 2004; Xie et al., 2006) based on the theory of Beylkin (1985), which combines detector and source information, focal beams analyze the detector and source parts of an acquisition geometry separately. Volker's formulations are applicable to homogeneous and heterogeneous media (Volker et al., 2001). Van Veldhuizen (2006) and Van Veldhuizen et al. (2008) developed a model-based implementation of the focal

\footnotetext{
Manuscript received by the Editor 11 October 2010; revised manuscript received 28 August 2011; published online 17 February 2012.

${ }^{1}$ Chinese Academy of Sciences, Institute of Geology and Geophysics, Key Laboratory of the Earth's Deep Interior, Beijing, China. E-mail: caswei@ gmail.com, lfu@mail.igcas.ac.cn.

${ }^{2}$ Delft University of Technology, Delphi Consortium, Delft, The Netherlands. E-mail: G.Blacquiere@tudelft.nl.

(C) 2012 Society of Exploration Geophysicists. All rights reserved.
} 
beam analysis method for inhomogeneous media by recursive depth extrapolation (Holberg, 1988) in the space-frequency domain.

Focal beam analysis is generally implemented in the frequency domain, and then the single-frequency focal beams can be simply extended to multifrequency focal beams by directly combining all the frequency components of band-limited seismic data (Van Veldhuizen et al., 2008). This straightforward approach involves a large calculation burden in the case of many frequencies and complex media, and hence, is often substantially restrained. In this paper, we propose a rapid method for the computation of 3D multifrequency focal beams in which a few single-frequency focal beam computations are followed by a number of interpolations for a range of frequencies and a practically defined seismic acquisition geometry. The 3D wavefield extrapolation in our focal beam computation is conducted by combined application of a 3D degenerate Fourier (DF) migrator and a 3D Born-Kirchhoff (BK) interpolation operator, which reduces the computational cost in complex media. The combined 3D DF-BK extrapolator was extended from the 2D case ( $\mathrm{Fu}, 2004 ; \mathrm{Fu}$ et al., 2006). It extrapolates wavefields through a thick slab and then interpolates wavefields in small layers. This approach has several advantages: (1) fast wavefield extrapolation through high-contrast media obtained by only using fast Fourier transforms (FFTs), (2) large-step extrapolation to further reduce computational costs, and (3) the BK interpolation accounting not only for obliquity, spherical-spreading, and wavelet-shaping factors, but also for the relative slowness perturbation in a laterally heterogeneous media. These advantages significantly improve the efficiency of the 3D multifrequency focal beam analysis that commonly involves extremely heavy computations. Finally, the multifrequency focal beam analysis is applied to a 3D model from an oil field in China, demonstrating how spatial sampling differences affect seismic imaging.

\section{MULTIFREQUENCY FOCAL BEAM}

The focal beam analysis method (Berkhout et al., 2001; Volker et al., 2001; Volker, 2002) originates from the migration of seismic reflection data using the common focus point concept (Berkhout, 1982; 1997). Seismic migration is the process of extracting the reflectivity information from the recorded seismic data. According to Berkhout et al. (2001), seismic migration corresponds to double focusing the seismic data according to

$$
\mathbf{P}\left(z_{m}, z_{m}\right)=\mathbf{F}\left(z_{m}, z_{0}\right) \mathbf{P}\left(z_{0}, z_{0}\right) \mathbf{F}\left(z_{0}, z_{m}\right),
$$

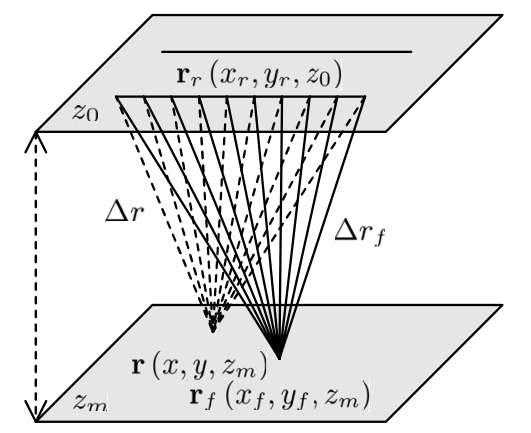

Figure 1. Schematic diagram of focal beams. Wave propagates form source grid-point at the target at $\mathbf{r}_{f}$ to detectors at the surface. The measured wave field is inverse-extrapolated to evaluate the focusing properties of the detector geometry at the target. where, $\mathbf{P}\left(z_{0}, z_{0}\right)=\mathbf{D}\left(z_{0}\right) \mathbf{W}\left(z_{0}, z_{m}\right) \mathbf{R}\left(z_{m}, z_{m}\right) \mathbf{W}\left(z_{m}, z_{0}\right) \mathbf{S}\left(z_{0}\right)$ is a frequency slice of the primary wavefield that is recorded at the surface at depth $z_{0}, \mathbf{D}\left(z_{0}\right)$ is the detector matrix, $\mathbf{S}\left(z_{0}\right)$ is the source matrix, $\mathbf{R}\left(z_{m}, z_{m}\right)$ is the reflectivity matrix containing angle-dependent reflectivity information, $\mathbf{F}\left(z_{0}, z_{m}\right)$ is the source-focusing matrix that removes wave propagation $\mathbf{W}\left(z_{m}, z_{0}\right)$ between the reflection level at depth $z_{m}$ and source level at depth $z_{0}, \mathbf{F}\left(z_{m}, z_{0}\right)$ is the detectorfocusing matrix that removes wave propagation $\mathbf{W}\left(z_{0}, z_{m}\right)$ between the reflection level at depth $z_{m}$ and detection level at depth $z_{0}$, and lateral coordinates $(\mathrm{x}, \mathrm{y})$ are implicit here. Note that the focusing matrices may include terms for correcting the imprint of the acquisition geometry in $\mathbf{D}\left(z_{0}\right)$ and $\mathbf{S}\left(z_{0}\right)$. Let us, for the moment, assume that such corrections are not implemented, such that in our analysis the imprint of the acquisition geometry becomes clear. In that case, because the focusing process is the reverse of the wave-propagation process, the focusing matrix is approximately equal to the conjugate complex of the propagation matrix (Berkhout, 1987).

We aim at analyzing the impact of acquisition geometry on the migration result and therefore assume the subsurface reflectivity to be unity

$$
\mathbf{R}\left(z_{m}, z_{m}\right)=\mathbf{I}\left(z_{m}, z_{m}\right),
$$

where $\mathbf{I}\left(z_{m}, z_{m}\right)$ is an identity matrix. In that case, the doublefocusing migration (equation 1) yields the following spatial resolution matrix

$$
\begin{aligned}
\mathbf{B}\left(z_{m}, z_{m}\right) & =\left[\mathbf{F}\left(z_{m}, z_{0}\right) \mathbf{D}\left(z_{0}\right) \mathbf{W}\left(z_{0}, z_{m}\right)\right]\left[\mathbf{W}\left(z_{m}, z_{0}\right) \mathbf{S}\left(z_{0}\right) \mathbf{F}\left(z_{0}, z_{m}\right)\right] \\
& =\mathbf{B}_{D}\left(z_{m}, z_{0}\right) \mathbf{B}_{S}\left(z_{0}, z_{m}\right),
\end{aligned}
$$

where $\mathbf{B}_{D}\left(z_{m}, z_{0}\right)$ and $\mathbf{B}_{S}\left(z_{0}, z_{m}\right)$ are the focal detector and focal source matrix, respectively. Note that in the ideal case $\mathbf{B}\left(z_{m}, z_{m}\right)$ equals $\mathbf{I}\left(z_{m}, z_{m}\right)$. However, in practice $\mathbf{B}\left(z_{m}, z_{m}\right)$ quantifies the imprint of the acquisition geometry on the reflectivity information (caused by the sparse sampling of the sources and detectors as given by $\mathbf{S}\left(z_{0}\right)$ end $\mathbf{D}\left(z_{0}\right)$, respectively).

According to Volker (2002), the continuous form of the spatial resolution function (equation 3) for a single temporal frequency component $\omega$ can be derived by considering the impact of a 3D target point $\mathbf{r}_{f}=\left(x_{f}, y_{f}, z_{m}\right)$ and a neighbor point $\mathbf{r}=\left(x, y, z_{m}\right)$ surrounding $\mathbf{r}_{f}$ (Figure 1)

$$
B\left(\mathbf{r}, \mathbf{r}_{f}, \omega\right)=B_{D}\left(\mathbf{r}, \mathbf{r}_{f}, \omega\right) B_{S}\left(\mathbf{r}, \mathbf{r}_{f}, \omega\right),
$$

where $B_{D}\left(\mathbf{r}, \mathbf{r}_{f}, \omega\right)$ and $B_{S}\left(\mathbf{r}, \mathbf{r}_{f}, \omega\right)$ are the focal detector and focal source beam, respectively. These are defined as

$$
\left\{\begin{array}{c}
B_{D}\left(\mathbf{r}, \mathbf{r}_{f}, \omega\right)=\int F\left(\mathbf{r}, \mathbf{r}_{f}\right) W\left(\mathbf{r}_{r}, \mathbf{r}_{f}\right) D\left(\mathbf{r}_{r}\right) d \mathbf{r}_{r} \\
B_{S}\left(\mathbf{r}, \mathbf{r}_{f}, \omega\right)=\int F\left(\mathbf{r}, \mathbf{r}_{s}\right) W\left(\mathbf{r}_{s}, \mathbf{r}_{f}\right) S\left(\mathbf{r}_{s}\right) d \mathbf{r}_{s}
\end{array}\right.
$$

$D\left(\mathbf{r}_{r}\right)$ is a detector sampling operator at $\mathbf{r}_{r}=\left(x_{r}, y_{r}, z_{0}\right) . S\left(\mathbf{r}_{s}\right)$ is a source sampling operator at $\mathbf{r}_{s}=\left(x_{s}, y_{s}, z_{0}\right) . F\left(\mathbf{r}, \mathbf{r}_{r}\right)$ describes inverse wavefield propagation from the detectors at $\mathbf{r}_{r}$ to all subsurface points $\mathbf{r}$ around the focal point $\mathbf{r}_{f} . W\left(\mathbf{r}_{r}, \mathbf{r}_{f}\right)$ describes forward wavefield propagation from the focal point $\mathbf{r}_{f}$ to all detectors $\mathbf{r}_{r}$. $F\left(\mathbf{r}, \mathbf{r}_{s}\right)$ describes inverse wavefield propagation from the sources at $\mathbf{r}_{s}$ to all subsurface points $\mathbf{r}$ around the focal point $\mathbf{r}_{f} . W\left(\mathbf{r}_{s}, \mathbf{r}_{f}\right)$ describes forward wavefield propagation from sources $\mathbf{r}_{s}$ to the focal point $r_{f}$.

Transforming the detector and source beams to the Radon domain, and extracting a horizontal section at $\tau=0$, Volker (2002) derived the amplitude versus ray parameter (AVP) function as 


$$
\tilde{B}\left(p_{x}, p_{y}\right)=\tilde{B}_{D}\left(p_{x}, p_{y}\right) \tilde{B}_{S}\left(-p_{x},-p_{y}\right)
$$

where, $p_{x}$ and $p_{y}$ are the lateral component of ray parameters, $\tilde{B}_{D}\left(p_{x}, p_{y}\right)$ and $\tilde{B}_{S}\left(p_{x}, p_{y}\right)$ are the Radon transforms of the detector and source beams, respectively.

Because seismic waves are never monochromatic, but always contain a frequency bandwidth, the single-frequency focal beams should be extended to multifrequency focal beams. In the following section, we present a rapid algorithm for the computation of multifrequency focal beams.

For 3D homogeneous and heterogeneous media, Volker (2002) introduced a simplified focal beam computation algorithm in the wavenumber and Radon domain, respectively. According to this author, the detector beam can be efficiently approximated by a Taylor series expansion, leading to the following expression making use of 2D spatial Fourier transforms for 3D homogeneous media

$$
B_{D}\left(\mathbf{r}, \mathbf{r}_{f}, \omega\right) \approx \frac{1}{4 \pi^{2}} \iint \exp \left[i\left(k_{x} \Delta x+k_{y} \Delta y\right)\right] D^{\prime}\left(k_{x}, k_{y}, \omega\right) d k_{x} d k_{y},
$$

where $k_{x}$ and $k_{y}$ are the horizontal wavenumber components, $\Delta x=x-x_{f}, \Delta y=y-y_{f}$ and $D^{\prime}\left(k_{x}, k_{y}, \omega\right)$ is the spatial Fourier transform of $D\left(\mathbf{r}_{r}\right)$ for the temporal frequency $\omega$.

For 3D heterogeneous media, the detector beam can be approximated by a Taylor series expansion, resulting in the following expression that makes use of 2D Fourier transforms in the 2D Radon domain

$$
\begin{aligned}
& B_{D}\left(\mathbf{r}, \mathbf{r}_{f}, \omega\right) \\
& \quad \approx \frac{\omega^{2}}{4 \pi^{2}} \iint \exp \left[i \omega\left(p_{x} \Delta x+p_{y} \Delta y\right)\right] D^{\prime \prime}\left(p_{x}, p_{y}, \omega\right) d p_{x} d p_{y},
\end{aligned}
$$

where $D^{\prime \prime}\left(p_{x}, p_{y}, \omega\right)$ is the Radon transform of $D\left(\mathbf{r}_{r}\right)$ for the temporal frequency $\omega$. Similar expressions also can be derived for the source beam and the spatial resolution function.

Volker (2002) developed equations 7 and 8 and established a rapid focal beam analysis algorithm in the wavenumber domain. Different from Volker (2002), we have endeavored to establish a conversion relationship between the focal beams under different frequencies using the same equations 7 and 8. Setting $d k_{x} d k_{y}=$ $k^{2} \frac{\left(z_{m}-z_{0}\right)^{2}}{\Delta r_{f}^{2}} d x_{r} d y_{r}$, equation 7 becomes

$B_{D}\left(\mathbf{r}, \mathbf{r}_{f}, \omega\right)$

$\approx \frac{k^{2}}{4 \pi^{2}} \iint \exp \left[i\left(k_{x} \Delta x+k_{y} \Delta y\right)\right] D^{\prime}\left(k_{x}, k_{y}, \omega\right) \frac{\left(z_{m}-z_{0}\right)^{2}}{\Delta r_{f}^{2}} d x_{r} d y_{r}$,

where, $k$ is the wavenumber, and $\Delta r_{f}=$ $\sqrt{\left(x_{f}-x_{r}\right)^{2}+\left(y_{f}-y_{r}\right)^{2}+\left(z_{m}-z_{0}\right)^{2}}$. Therefore, the focal detector beams under different frequencies satisfy, within a degree of confidence, the following relationship for homogeneous and heterogeneous media

$$
B_{D}\left(\Delta x, \Delta y, \mathbf{r}_{f}, c \omega\right) \approx \frac{1}{c^{2}} B_{D}\left(c \Delta x, c \Delta y, \mathbf{r}_{f}, \omega\right)
$$

where $c$ is a constant $(0<c<1), \Delta x=x-x_{f}$, and $\Delta y=y-y_{f}$.

According to equation 10, the focal detector beam at frequency $c \omega$ can be derived directly from the focal detector beam at frequency $\omega$ via interpolation. Similar to the focal detector beams, the relationship for the focal source beams and the combined focal beams (resolution function and AVP function) for different frequencies can also be approximated as follows

$$
\begin{array}{r}
B_{S}\left(\Delta x, \Delta y, \mathbf{r}_{f}, c \omega\right) \approx \frac{1}{c^{2}} B_{S}\left(c \Delta x, c \Delta y, \mathbf{r}_{f}, \omega\right), \\
B\left(\Delta x, \Delta y, \mathbf{r}_{f}, c \omega\right) \approx \frac{1}{c^{2}} B\left(c \Delta x, c \Delta y, \mathbf{r}_{f}, \omega\right) .
\end{array}
$$

It indicates that focal beams for a frequency range can be approximated by conducting a single-frequency focal beam analysis and compute the other frequencies via interpolation operations (see Figure 2). Different from the directional illumination analysis using beamlet decomposition and propagation (Wu and Chen, 2006), the multifrequency focal beam (Figure 2) cannot be obtained by directly summing frequencies. Therefore, we use all the frequency components of the focal beams to carry out an inverse Fourier transform to the time domain and extract the $t=0$ slice as the result of the multifrequency focal beam. If $\mathrm{N}$ is the number of frequency components, this approach is roughly $N$ times faster than the direct calculation of each frequency component because the computational cost is heavily weighted on the wavefield simulation. If needed, we can always increase the accuracy of the method by computing more single-frequency beams and interpolate less - at the cost of computational efficiency.

\section{DEGENERATE FOURIER WAVEFIELD EXTRAPOLATION PLUS BORN-KIRCHHOFF WAVEFIELD INTERPOLATION}

To implement focal beam analysis, equation 5 needs to be evaluated. In doing this, we should choose a method to propagate waves, as accurately as possible, for the estimation of the forward propagation operators $W$ and the focusing operators $F$. The computational burden for the multifrequency focal beam analysis depends greatly on the efficiency of the chosen method for numerical wave propagation. To achieve an acceptable trade-off between numerical accuracy and computation time for given computer resources, we have developed a method for fast numerical wave propagation that combines a $3 \mathrm{D}$ DF operator with a $3 \mathrm{D}$ BK wavefield interpolation. This DF-BK method can significantly enhance

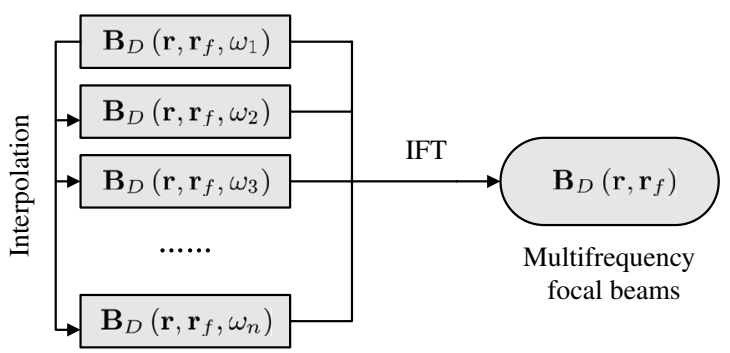

Figure 2. An efficient algorithm for multifrequency focal beams. According to equation 10, the focal beams at a frequency of $\omega_{2}$, $\omega_{3}$, and $\omega_{n}$ can be derived directly by interpolating the focal beams at a frequency of $\omega_{1}$. Then the focal beams for a given frequency range can be derived approximately by executing one singlefrequency focal beam analysis with interpolation operations. An inverse Fourier transform is applied to convert all the frequency components into time domain. 
the efficiency of 3D multifrequency focal beam analyses for complex media.

\section{One-way Lippmann-Schwinger integral equation}

The 3D DF-BK propagator can be derived from the generalized Lippmann-Schwinger integral equation ( $\mathrm{Fu}$ et al., 1997) which is equivalent to the Helmholtz equation and describes two-way wave propagation in heterogeneous media. To formulate one-way approximations of the generalized Lippmann-Schwinger integral equation, we slice heterogeneous media horizontally into a stack of heterogeneous slabs. Similar to the 2D case (Fu, 2006), applying the plane-wave representation of the Hankel function to the generalized Lippmann-Schwinger integral equation, the 3D one-way Lippmann-Schwinger integral equation can be formulated inside a heterogeneous slab (Figure 3)

$$
\begin{array}{r}
\left(k_{z}+k_{z}^{\prime}\right) u\left(k_{x}, k_{y}, z+\Delta z\right)-\frac{k_{0}}{2} F_{x y}\left(k_{x}, k_{y}, z+\Delta z\right) \\
=\left[2 k_{z} u\left(k_{x}, k_{y}, z\right)+\frac{k_{0}}{2} F_{x y}\left(k_{x}, k_{y}, z\right)\right] \exp \left(i k_{z} \Delta z\right),
\end{array}
$$

where $u$ is the seismic displacement vector, $k_{x}, k_{y}$ and $k_{z}$ are the components of the wavenumber, $k_{z}^{\prime}$ is the vertical wavenumber of neighboring media below the slab, $k_{0}=\omega / v_{0}$ is the reference wave number, $\omega$ which is the radian frequency, and $v_{0}$ is the reference velocity, and $F_{x y}\left(k_{x}, k_{y}, z\right)=\mathrm{FT}_{x y}\left\{i k_{0}[n(\mathbf{r})-1] u(\mathbf{r})\right\}$ with $\mathrm{FT}_{x y}$ being the horizontally oriented 2D Fourier transform, and $n(\mathbf{r})=v_{0} / v(\mathbf{r})$ being the refraction index of an acoustic wave. One-way Lippmann-Schwinger integral equation accounts for the accumulated effect of forward scattering by volume heterogeneities inside a slab and the transmission/refraction between different slabs on wave amplitude and phase.

Equation 13 can be simplified into the following form by using the Born approximation (refer to Appendix A for details)

$$
\begin{aligned}
& u\left(k_{x}, k_{y}, z+\Delta z\right) \\
& \quad=\left[u\left(k_{x}, k_{y}, z\right)+\frac{1}{\bar{k}_{z}} F_{x y}\left(k_{x}, k_{y}, z\right)\right] \exp \left(i k_{z} \Delta z\right) .
\end{aligned}
$$

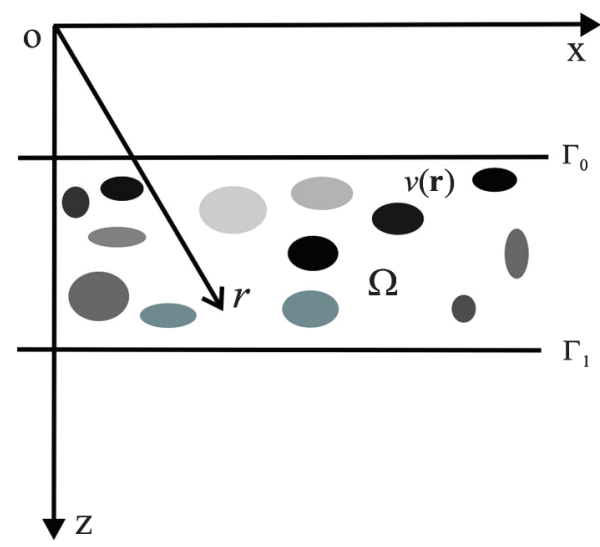

Figure 3. The geometry of a heterogeneous slab with the thickness $\Delta z . \mathbf{r}$ is the position vector and $v(\mathbf{r})$ is the velocity. The slab occupying a region $\Omega$ is bounded by the upper boundary $\Gamma_{0}$ and the lower boundary $\Gamma_{1}$.
The dispersion relation corresponding to equation 14 is

$$
\bar{k}_{z}=\sqrt{1-\bar{k}_{x}^{2}-\bar{k}_{y}^{2}}+(n-1)\left(1-\bar{k}_{x}^{2}-\bar{k}_{y}^{2}\right)^{-1 / 2},
$$

where we normalize the wavenumbers by $\bar{k}_{x}=k_{x} / k_{0}, \bar{k}_{y}=k_{y} / k_{0}$, and $\bar{k}_{z}=k_{z} / k_{0}$, and $n$ is the different constants of the refractive index $n(\mathbf{r})$ to avoid the convolution operation.

\section{D DF operator}

Using $\bar{k}_{x} \leq 1$ and $\bar{k}_{y} \leq 1$ (Huang et al., 1999) for postcritical wave propagation, the term $\left(1-\bar{k}_{x}^{2}-\bar{k}_{y}^{2}\right)^{-1 / 2}$ can be approximated as follows

$$
\left(1-\bar{k}_{x}^{2}-\bar{k}_{y}^{2}\right)^{-1 / 2}=1-\sum_{j=1}^{\infty} \frac{a_{j}\left(\bar{k}_{x}^{2}+\bar{k}_{y}^{2}\right)}{1+b_{j}\left(\bar{k}_{x}^{2}+\bar{k}_{y}^{2}\right)},
$$

where the coefficients $a_{j}$ and $b_{j}$ are independent of the refraction index $n$. Then, we have

$$
\begin{aligned}
\bar{k}_{z}= & \sqrt{1-\bar{k}_{x}^{2}-\bar{k}_{y}^{2}}+(n-1) \\
& -(n-1) \sum_{j=1}^{\infty} \frac{a_{j}\left(\bar{k}_{x}^{2}+\bar{k}_{y}^{2}\right)}{1+b_{j}\left(\bar{k}_{x}^{2}+\bar{k}_{y}^{2}\right)} .
\end{aligned}
$$

Equation 17 is a DF operator (or separation-of-variables operator) that leads to a pure Fourier-transform-based matching solution for wavefield extrapolation (Fu et al., 2006 for the 2D case). The coefficients $a_{j}$ and $b_{j}$ in equation 17 are determined numerically by a least-squares optimization procedure by which optimum coefficients can be searched for that minimize frequency dispersion errors.

Because of the mathematical properties and approximation behavior of rational functions (Trefethen and Halpern, 1986; Bamberger et al., 1988), equation 17 should be well-posed theoretically, especially for lower-order terms. Equation 17 with an expansion of infinite series needs to be truncated for numerical computations. In practice, the first-order equation or, at most, the second-order equation is adequate for common one-way wave propagation in large-contrast media with large propagation angles (see more detail in the following section).

Using the exponential approximation $e^{i \xi} \approx 1+i \xi$, the regional separation-of-variables operator expressed by equation 17 yields the following one-way propagation equation

$$
\begin{aligned}
u\left(k_{x}, k_{y}, z+\Delta z\right) & \\
= & {\left[\sum_{j=1}^{\infty} \frac{a_{j}\left(\bar{k}_{x}^{2}+\bar{k}_{y}^{2}\right)}{1+b_{j}\left(\bar{k}_{x}^{2}+\bar{k}_{y}^{2}\right)}+\left(1-\sum_{j=1}^{\infty} \frac{a_{j}\left(\bar{k}_{x}^{2}+\bar{k}_{y}^{2}\right)}{1+b_{j}\left(\bar{k}_{x}^{2}+\bar{k}_{y}^{2}\right)}\right)\right.} \\
& \left.\quad \exp \left[i k_{0} \Delta z(n-1)\right]\right] u\left(k_{x}, k_{y}, z\right) \exp \left(i k_{0} \Delta z\right) .
\end{aligned}
$$

Setting $C_{j}=a_{j}\left(\bar{k}_{x}^{2}+\bar{k}_{y}^{2}\right) /\left[1+b_{j}\left(\bar{k}_{x}^{2}+\bar{k}_{y}^{2}\right)\right]$ and taking the firstorder approximation, equation 18 becomes

$$
\begin{aligned}
& u\left(k_{x}, k_{y}, z+\Delta z\right) \\
& \quad=\left\{C_{1}+\left(1-C_{1}\right) \mathrm{FT}_{x y}\left[u\left(k_{x}, k_{y}, z\right) \exp \left(i k_{0} \Delta z\right)\right]\right\} .
\end{aligned}
$$


We see that the first-order DF operator is actually a linear interpolation in the wavenumber domain between two split-step terms. It significantly improves on the split-step Fourier (SSF) method (Stoffa et al., 1990) for large lateral variations at the cost of one more Fourier transform in each slab. The accuracy of the DF method will be discussed in detail in a following section.

\section{D BK operator for wavefield interpolation}

One-way propagation by equation 14 using Fourier transforms is actually unstable because of the singularity that occurs when $\bar{k}_{z} \approx 0$ (large-angle waves). However, one-way propagation using its space-domain version is unconditionally stable using diffraction summation (Fu, 2004 for the 2D case). The space-domain version of equation 14 can be obtained using Fourier transforms

$$
\begin{aligned}
& u\left(k_{x}, k_{y}, z+\Delta z\right) \\
& \quad=\int \frac{i k_{0}}{2 \pi \Delta r} \exp \left(i k_{0} \Delta z(n-1)\right) \exp \left(i k_{0} \Delta r\right) u\left(k_{x}, k_{y}, z\right) d x d y,
\end{aligned}
$$

where $\Delta r=\sqrt{\left(x_{1}-x_{2}\right)^{2}+\left(y_{1}-y_{2}\right)^{2}+\Delta z^{2}}$ and each downwardcontinued output trace at $\left(x_{2}, y_{2}\right)$ is calculated by integration over the input traces at $\left(x_{1}, y_{1}\right)$. This space-domain diffraction formulation is a BK integral expression, which differs from the traditional Kirchhoff propagator,

$$
u\left(k_{x}, k_{y}, z+\Delta z\right)=\int \frac{i k_{0}}{2 \pi \Delta r} \cos \theta \exp \left(i k_{0} \Delta r\right) u\left(k_{x}, k_{y}, z\right) d x d y
$$

$\theta$ being the angle between the normal to the input interface and the traveltime path, in that it accounts not only for the obliquity, spherical-spreading, and wavelet-shaping factors, but also for relative slowness perturbations in laterally heterogeneous media. The accuracy of 3D BK operator will be discussed in detail in the following section.

\section{Accuracy analysis of DF and BK operators}

Using a small-angle assumption $\left(1-\bar{k}_{x}^{2}-\bar{k}_{y}^{2}\right)^{-1 / 2}$ in equation 15 yields the following SSF dispersion equation

$$
\bar{k}_{z}=\sqrt{1-\bar{k}_{x}^{2}-\bar{k}_{y}^{2}+(n-1)}
$$

For strongly contrasting media, various hybrid methods that incorporate the FD scheme into the Fourier matching solutions have been proposed, for example, the split-step FD propagator (Thomson, 1990), the split-step Padé solution (Collins, 1993), and the Fourier finite-difference migrator (FFD) (Ristow and Ruhl, 1994). These rational approximations to the square-root function dispersion relation can be generally expressed as

$$
\bar{k}_{z}=\sqrt{1-\bar{k}_{x}^{2}-\bar{k}_{y}^{2}}+(n-1)-\sum_{j=1}^{\infty} \frac{a_{j}(n)\left(\bar{k}_{x}^{2}+\bar{k}_{y}^{2}\right)}{1+b_{j}(n)\left(\bar{k}_{x}^{2}+\bar{k}_{y}^{2}\right)},
$$

with its coefficients $a_{j}(n)$ and $b_{j}(n)$ varying with the refraction index $n$. We see that equation 23 hierarchically consists of a reference phase-shift solution (the first term), a split-step correction term (the second term), and a parabolic correction term (the last term). The crosscoupling of $k_{z}$ and $n$ in the last term shows that equation 22 is not a degenerative operator expression and consequently requires an extra implicit FD implementation. The FFD method, despite its high numerical accuracy for strong velocity variations, causes grid dispersion, 3D splitting error, and computational burden. In contrast, DF operator with constant coefficients $a_{j}$ and $b_{j}$ independent of $n$ in equation 17 leads to a pure Fourier-transform-based matching solution for wavefield extrapolation.

To conduct a quantitative assessment of the DF and BK operators, Figure 4 shows the angular spectra of the first-order DF operator and the BK operator, as compared with those of the FFD and SSF propagators under a $10 \%$ relative phase error. As expected, the first-order DF operator is accurate for all values of the acoustic refraction index, almost approaching the accuracy of the FFD. The accuracy of the BK operator is between SSF and DF, implying an acceptable application for wavefield interpolation in small layers inside a thick extrapolated slab. This accuracy comparison of the first-order DF propagators with other Fourier propagators demonstrates a quick convergence of the regional DF approximation in the low-order terms. Furthermore, the extrapolation operators have been designed for a maximum wavepropagation angle of $60^{\circ}$. Because the DF method only uses Fourier transforms for propagation and migration of wavefields, the grid dispersion and 3D splitting error (Zhang et al., 2007) that occurs with the 3D FFD method can be avoided.

\section{Implementation of multifrequency focal beam analysis by the DF-BK operator}

Computation of focal beams consists of the following steps.

- Select a grid point at the target in the subsurface.

- Simulate the angle-independent grid-point response by the DF-BK propagator from this grid point to the acquisition

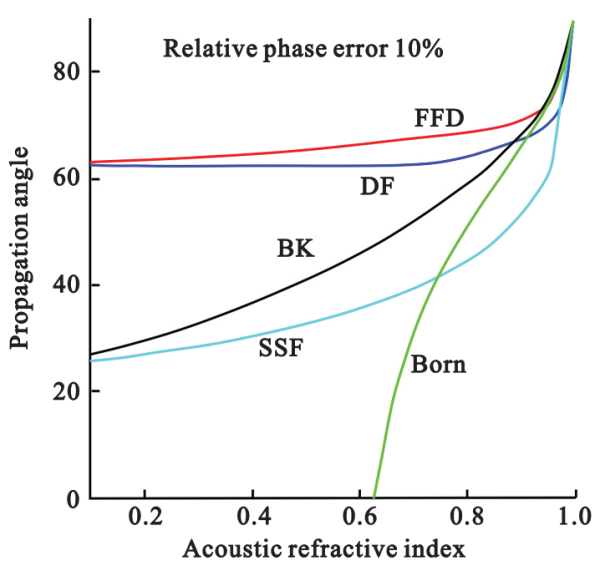

Figure 4. Comparison of angular spectra of various dispersion relations: the Born, the Born-Kirchhoff (BK), the split-step Fourier (SSF), the Degenerate Fourier (DF), and the Fourier finitedifference (FFD) under a $10 \%$ relative phase error $(\mathrm{Fu}, 2006)$. 


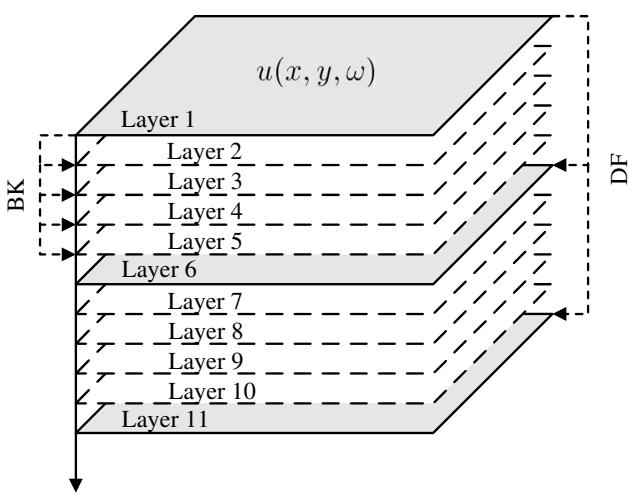

Figure 5. Schematic diagram of the DF-BK propagator. We first conduct a large-step extrapolation of wavefields through a thick slab by the DF propagator using equation 19 , and then interpolate the extrapolation results in small heterogeneous layers inside the slab by the BK operator using equation 20. $u(x, y, z)$ is the seismic displacement vector with $\omega$ is the radian frequency.
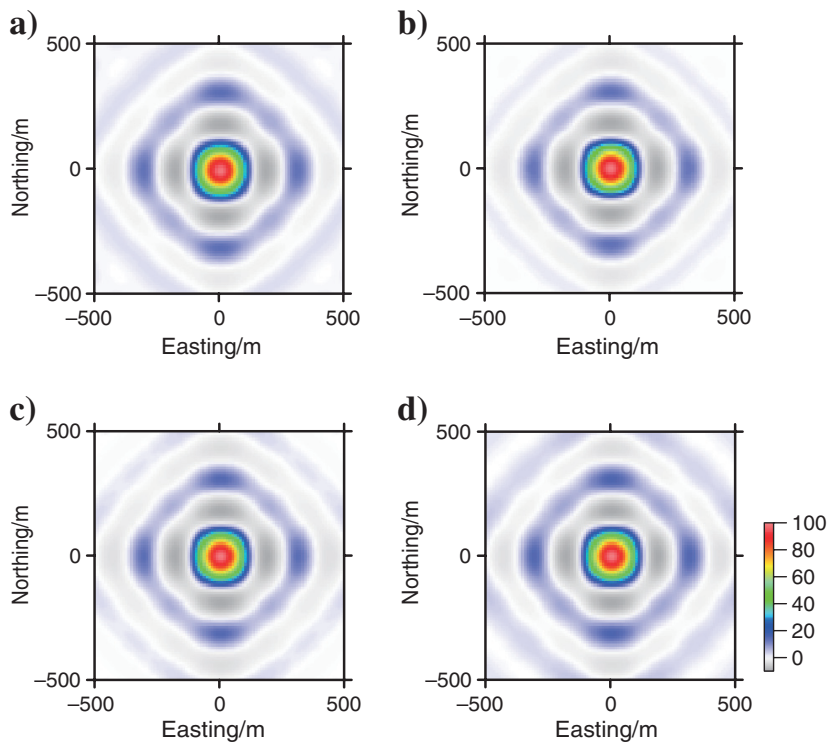

Figure 6. Detector beam in homogeneous media at $10 \mathrm{~Hz}$ (a) directly calculated using equation 4 , and, using equation 10 , (b) obtained from the $20 \mathrm{~Hz}$ beam, (c) the $30 \mathrm{~Hz}$ beam, and (d) and the $40 \mathrm{~Hz}$ beam. The colors indicate the amplitudes on a linear scale. level. As shown in Figure 5, we first conduct a large-step extrapolation of the wavefield through a thick slab by the DF propagator using equation 19, and then interpolate the large-step extrapolation results in small heterogeneous layers inside the slab by the BK operator using equation 20 .

- Apply the acquisition geometry by selecting traces according to: (i) source locations for the computation of focal source beams and (ii) detector locations for the computation of focal detector beams.

- Apply the focusing from the acquisition level to a volume around the target point using: (i) the inverse wavefield extrapolator $F\left(\mathbf{r}, \mathbf{r}_{r}\right)$ in equation 5 for focusing on the detector side and (ii) the inverse wavefield extrapolator $F\left(\mathbf{r}, \mathbf{r}_{s}\right)$ in equation 5 for focusing on the source side.

- Substitute the detector and source focal beams in equations 5 into equations 4 and 6 to compute the spatial resolution and AVP imprints.

- Interpolate the single-frequency focal beams (see Figure 2) to a frequency range by equation 12 and sum all the frequency components.

To avoid the repetition of wavefield simulations for forward and backward extrapolations, we first apply the wavefield simulation to the deepest target layer by the DF-BK propagator, and then extract data for focal beam analysis. This approach is relatively efficient because the computational cost is heavily weighted on the wavefield simulation. Note that the evaluated volume around a target point should be sufficiently large to include the grid-point locations at which the main imaging artifacts such as spatial aliasing may occur (Van Veldhuizen et al., 2008).

\section{EXAMPLES}

\section{Focal beams for individual frequencies}

This section presents an example of detector beams for a landbased seismic acquisition geometry. The geometry consists of eight detector lines, each $1500 \mathrm{~m}$ in length, with $200 \mathrm{~m}$ spacing between lines and a $100 \mathrm{~m}$ interval between detector points along the line. The geometry template is not rolled along during the acquisition. The target point is located at a depth of $1000 \mathrm{~m}$ below the center of the spread. The detector beam for a frequency of $10 \mathrm{~Hz}$ are directly calculated under a homogeneous medium condition using equation 5, with the result shown in Figure 6a. For reference, the synthetic results for a frequency of $10 \mathrm{~Hz}$, calculated from detector beams of 20,30, and $40 \mathrm{~Hz}$, are shown in Figure 6b, 6c, and
Figure 7. The revised 3D SEG/EAGE Salt Model (Aminzadeh et al., 1997) with total dimensions of $2.5 \mathrm{~km}(\mathrm{x}) \times 2.5 \mathrm{~km}(\mathrm{y}) \times 1 \mathrm{~km}(\mathrm{z})$. Grid spacing is $10 \mathrm{~m}$ for both lateral directions and $5 \mathrm{~m}$ in the vertical direction. The target point is located at the point $(1250,1250,500)$ near the salt bottom (black star in right images). Velocities vary from $1500 \mathrm{~m} / \mathrm{s}$ in the upper layer to $4482 \mathrm{~m} / \mathrm{s}$ in the bottom layer. The geometry consists of eight detector lines (white line in left image), each $1500 \mathrm{~m}$ in length, with $200 \mathrm{~m}$ spacing between lines and a $100 \mathrm{~m}$ interval between detector points along the line. a)

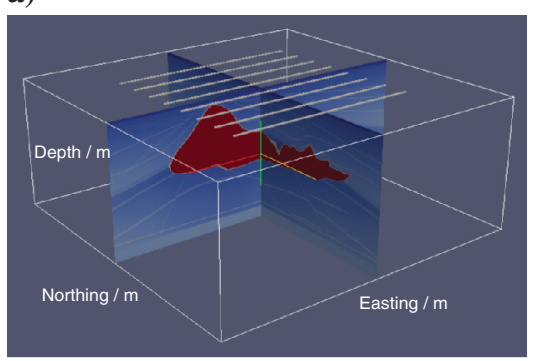

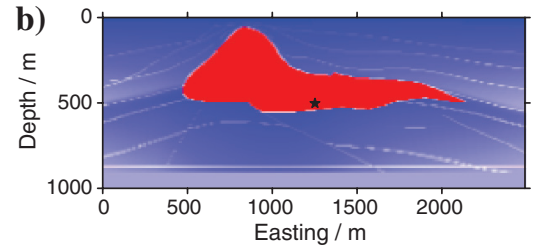

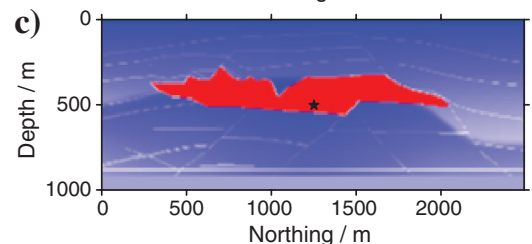


$6 \mathrm{~d}$, respectively. These figures show that for a homogeneous medium the focal beam obtained from the direct calculation is almost identical to those computed from other single-frequency beams. This example confirms that the approximation by equation 7 has a high accuracy for a homogeneous medium.

The revised 3D SEG/EAGE Salt Model (Aminzadeh et al., 1997; see Figure 7) with total dimensions of $2.5 \mathrm{~km}(\mathrm{x}) \times 2.5 \mathrm{~km}(\mathrm{y}) \times$ $1 \mathrm{~km}(\mathrm{z})$ is taken as a complex example of a heterogeneous medium assemblage. We reduced the grid spacings to $10 \mathrm{~m}$ for both lateral directions and $5 \mathrm{~m}$ for the vertical direction, making them eight times smaller than the original size. The drastic changes of the velocity with a smaller grid spacing cause a more complex model than the original one. The same acquisition geometry used in the previous example also has been chosen for this model. The target point is located at $(x, y, z)=(1250,1250,500) \mathrm{m}$, near the salt bottom (black star in right image of Figure 7). Velocities vary from $1500 \mathrm{~m} / \mathrm{s}$ in the upper layer to $4482 \mathrm{~m} / \mathrm{s}$ in the salt. The detector beam at $10 \mathrm{~Hz}$ is calculated directly by the DF-BK propagator assuming heterogeneous media conditions, with the result shown in Figure 8a. Note that the undersampling of detectors leads to apparent spatial aliasing in the $y$-direction. Similarly, the synthetic results, obtained from the detector beams at 20,30 , and $40 \mathrm{~Hz}$ using equation 8, are shown in Figure 8b, 8c, and 8d, respectively. We can see that the results obtained by both the direct calculation and the computation using other single-frequency beams are similar for heterogeneous media, especially in the main lobe, but with some differences in the side lobes because of the sensitivity differences of the 20,30, and $40 \mathrm{~Hz}$ beams to local variations in the model. The agreement between the direct calculation and interpolation confirms the validity of the interpolation technique for heterogeneous media, even in this complex model. Consequently, we can apply the approach to multifrequency focal beam.

\section{Focal beams for a range of frequencies}

The acquisition geometry and velocity model of the previous example is used again. Figure 9b shows the multifrequency detector beam for $10-40 \mathrm{~Hz}$ with a dominant frequency is $25 \mathrm{~Hz}$ obtained by interpolation according to equation 8 using the single-frequency result (see Figure 9a) at $40 \mathrm{~Hz}$ for a homogeneous medium. For reference, Figure 9c shows the synthetic result for $10-40 \mathrm{~Hz}$ obtained by directly summing frequencies. Figure $9 \mathrm{~d}, 9 \mathrm{e}$, and $9 \mathrm{f}$ show the same beams for a heterogeneous medium. Similar to the single-frequency results (Figure 9a, 9b, and 9c), there is apparent spatial aliasing in the $y$-direction in the multifrequency focal beams (Figure 9d, 9e, and 9f). As expected, the multifrequency focal beam, shown in Figure 9b, 9c, 9e, and 9f, due to its larger temporal frequency range than the single-frequency focal beams shown in Figure 9a and 9d, are characterized by a higher spatial resolution despite its lower dominant frequency. In addition, the side-lobe energy distribution of the multifrequency focal beams is more diffuse than that of the single-frequency focal beams because of destructive interference effects of the various frequencies involved. The agreements between Figure $9 \mathrm{~b}$ and $9 \mathrm{c}$ or Figure $9 \mathrm{e}$ and $9 \mathrm{f}$
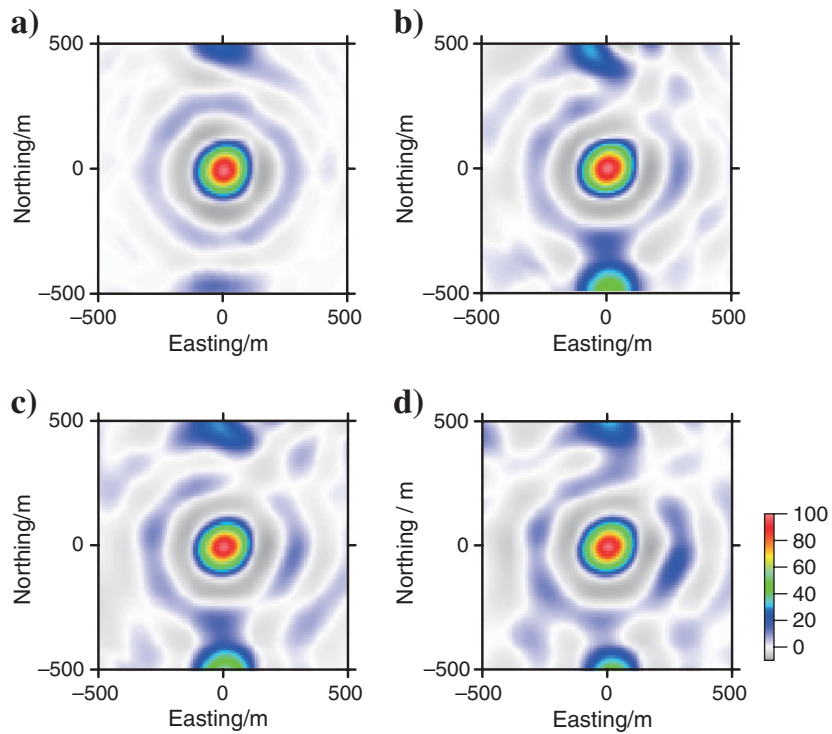

Figure 8. Detector beam in heterogeneous media at $10 \mathrm{~Hz}$ (a) directly calculated using equation 4 , and using equation 10 , (b) obtained from the $20 \mathrm{~Hz}$ beam, (c) the $30 \mathrm{~Hz}$ beam, and (d) the $40 \mathrm{~Hz}$ beam. The colors indicate the amplitudes on a linear scale. a)

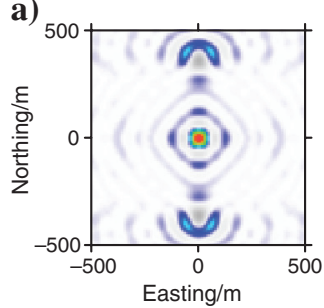

b)

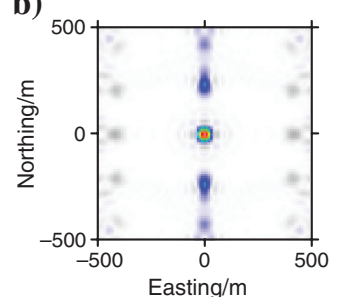

c)
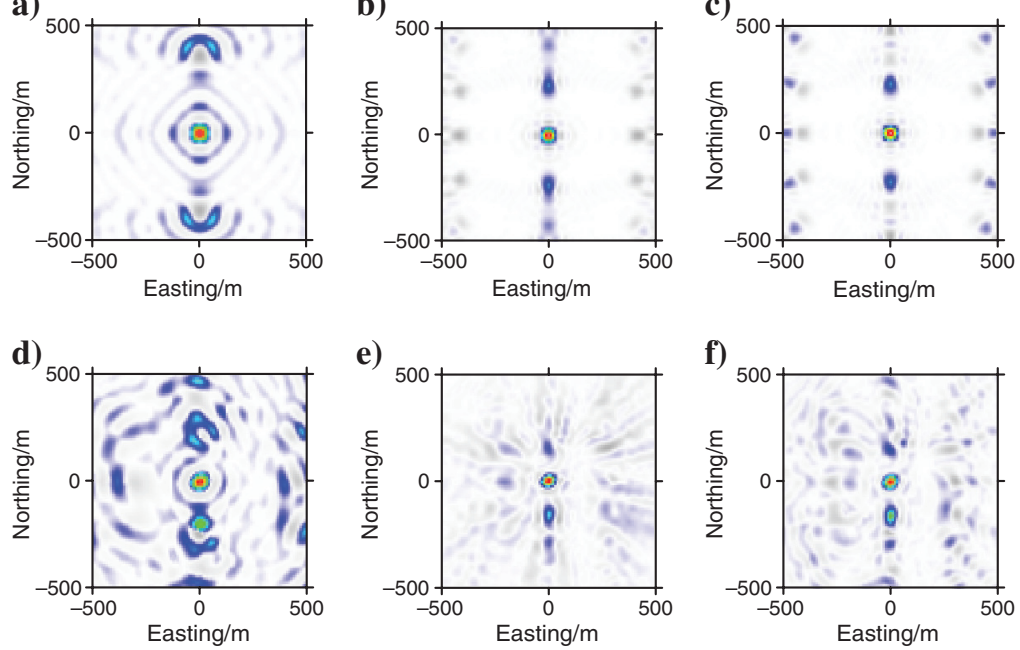

e)

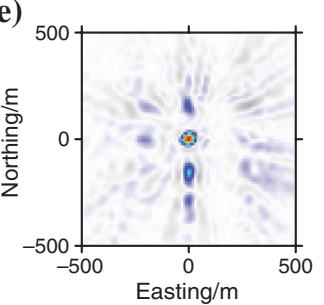

f)

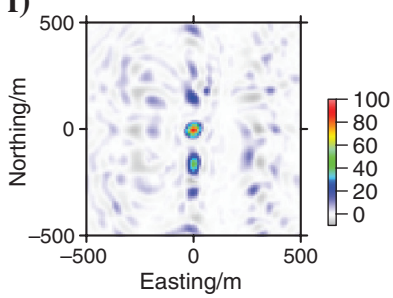

Figure 9. Single- and multifrequency detector beams. (a) Detector beam at $40 \mathrm{~Hz}$ in homogeneous medium, (b) Detector beam for $10-40 \mathrm{~Hz}$ by interpolation at $40 \mathrm{~Hz}$ in homogeneous media, (c) The sum of single-frequency detector beams for $10-40 \mathrm{~Hz}$ in homogeneous medium, (d) Detector beam at $40 \mathrm{~Hz}$ in heterogeneous medium, (e) Detector beam for $10-40 \mathrm{~Hz}$ by interpolation at $40 \mathrm{~Hz}$ in heterogeneous medium, and (f) The sum of single-frequency detector beams for $10-40 \mathrm{~Hz}$ in heterogeneous medium. The colors indicate the amplitudes on a linear scale. 
demonstrate that the multifrequency focal beam analysis proposed in this article can be extended to any frequency range through single-frequency interpolation without greatly increasing computational cost.

a)
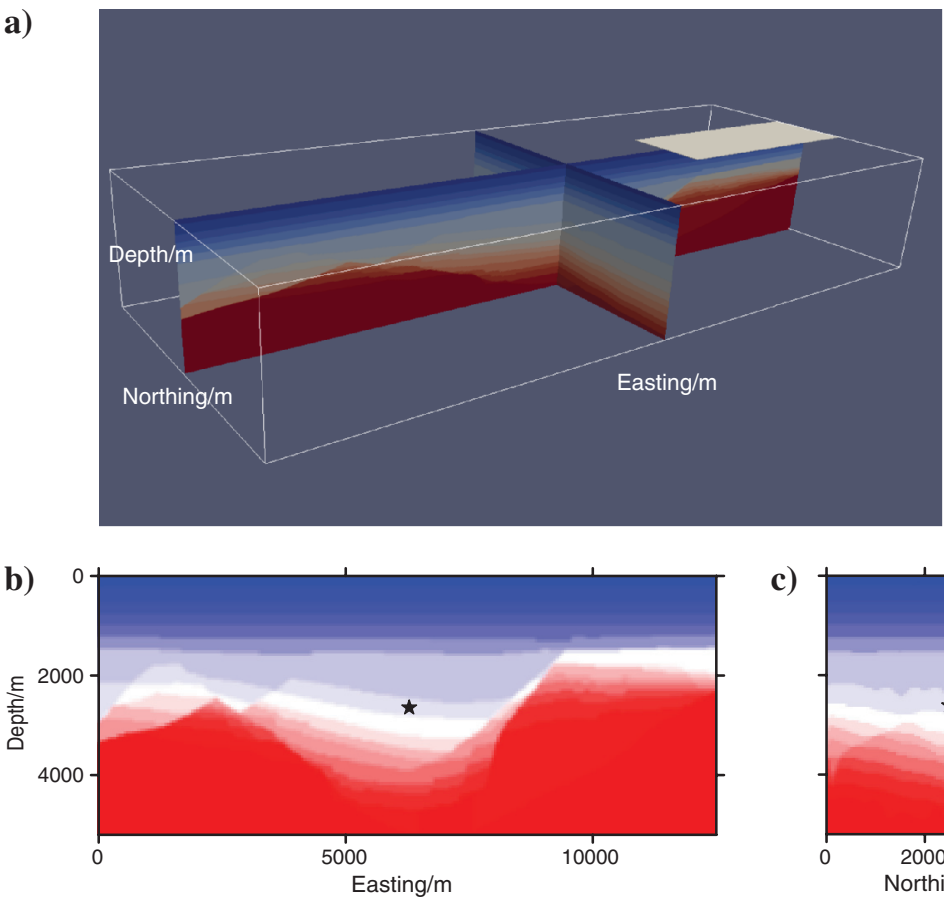

c)

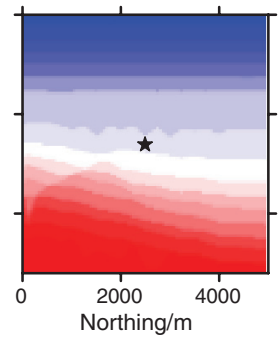

Figure 10. 3D real velocity model of an oilfield in China. The model with total dimensions of $12.5 \mathrm{~km}(\mathrm{x}) \times 5 \mathrm{~km}(\mathrm{y}) \times 5.2 \mathrm{~km}(\mathrm{z})$, contains a number of layers with complex fault structures. Velocities vary from $2100 \mathrm{~m} / \mathrm{s}$ in the upper layer to $5500 \mathrm{~m} / \mathrm{s}$ in the bottom layer. Grid spacing in the model is $25 \mathrm{~m}$ for lateral and vertical directions. The target point is located at the point $(3125,1250,2313)$ (black star in bottom images). The geometries consist of 16 detector lines (white rectangle in top image).

\section{Focal beam analysis for a real model}

In this section, we examine an example of a real 3D model from an oilfield in China. The model, shown in Figure 10 with total dimensions of $12.5 \mathrm{~km}(\mathrm{x}) \times 5 \mathrm{~km}(\mathrm{y}) \times 5.2 \mathrm{~km}(\mathrm{z})$, contains a number of layers with complex fault structures. Velocities vary from $2100 \mathrm{~m} / \mathrm{s}$ in the upper layer to $5500 \mathrm{~m} / \mathrm{s}$ in the bottom layer. Grid spacing in the model is $25 \mathrm{~m}$ for both lateral directions and $10 \mathrm{~m}$ for the vertical direction. The target point is located at the point $(3125$, 1250, 2313). Two orthogonal acquisition templates, corresponding to acquisition Scheme I and Scheme II, respectively, shown in Figure 11, were adopted with the detailed parameters listed in Table 1 . The geometry templates were rolled 20 times longitudinally and three times transversely during the acquisition. Four spread cables were rolled transversely each time with full fold being 160. The dominant frequency used in the analysis is $20 \mathrm{~Hz}$.

To reduce computational cost, we first performed wavefield simulations for the whole model using the DF-BK propagator, and then extracted data for focal beam analysis. The multifrequency focal beam analyses were conducted for the frequency range $10-35 \mathrm{~Hz}$ for Schemes I and II (see Figure 11). The resulting focal detector and source beams and their Radon-domain versions, the spatial resolution function, and the AVP imprints are shown in Figures 12 (Scheme I) and 13 (Scheme II) for a hydrocarbon target depth of $2313 \mathrm{~m}$. We see that the main lobes in the detector beams, the source beams and the spatial resolution function are almost identical for these two schemes in the space domain, implying they have a similar spatial resolution. The aliasing effects along the $x$-direction which don't appear on Scheme I are especially heavy in Scheme II for the reason of the profound difference of the detector point spacing. Scheme I shows a more uniform range of the AVP imprints for the $x$-direction and has a more concentrated energy response in the spatial resolution function with smaller side lobes. The smaller detector interval used in Scheme I, also make the angle-dependent amplitude information at the target point in the $x$-direction more uniform. Therefore, Scheme I may be more favorable for seismic imaging and amplitude inversion. In conclusion, with increasing channel intervals, as expected, the spatial resolution of the acquisition geometry is not affected, but the side-lobe energy increases and the AVP imprint for the $x$-direction becomes more nonuniform.

Multifrequency focal beams for the frequency range $10-35 \mathrm{~Hz}$ were computed for Schemes I and II on an Intel Xeon X5355 processor with a $2.66 \mathrm{GHz}$ CPU, and $16 \mathrm{~GB}$ RAM. The CPU run time for the $3 \mathrm{D}$ model with $501 \times 201 \times 209$ grid blocks was about 8 hours.

\section{DISCUSSION}

In the previous section, we discussed fast multifrequency focal beam analysis of seismic acquisition geometries. The method aims to assess the image quality for a given acquisition geometry 
Table 1. Parameters of two acquisition geometry (Scheme I and II).

\begin{tabular}{|c|c|c|c|c|c|c|c|c|c|}
\hline Survey & Geometry & $\begin{array}{c}\text { Detector } \\
\text { point } \\
\text { distance }\end{array}$ & $\begin{array}{c}\text { Source } \\
\text { point } \\
\text { distance }\end{array}$ & $\begin{array}{l}\text { Detector } \\
\text { line } \\
\text { distance }\end{array}$ & $\begin{array}{c}\text { Source } \\
\text { line } \\
\text { distance }\end{array}$ & Bin size & Folds & $\begin{array}{c}\text { Inline } \\
\text { rolling } \\
\text { distance }\end{array}$ & $\begin{array}{c}\text { Crossline } \\
\text { rolling } \\
\text { distance }\end{array}$ \\
\hline Scheme II & 16L3S40R & $100 \mathrm{~m}$ & $100 \mathrm{~m}$ & $100 \mathrm{~m}$ & $100 \mathrm{~m}$ & $50 \mathrm{~m} \times 50 \mathrm{~m}$ & 160 & $100 \mathrm{~m}$ & $400 \mathrm{~m}$ \\
\hline
\end{tabular}
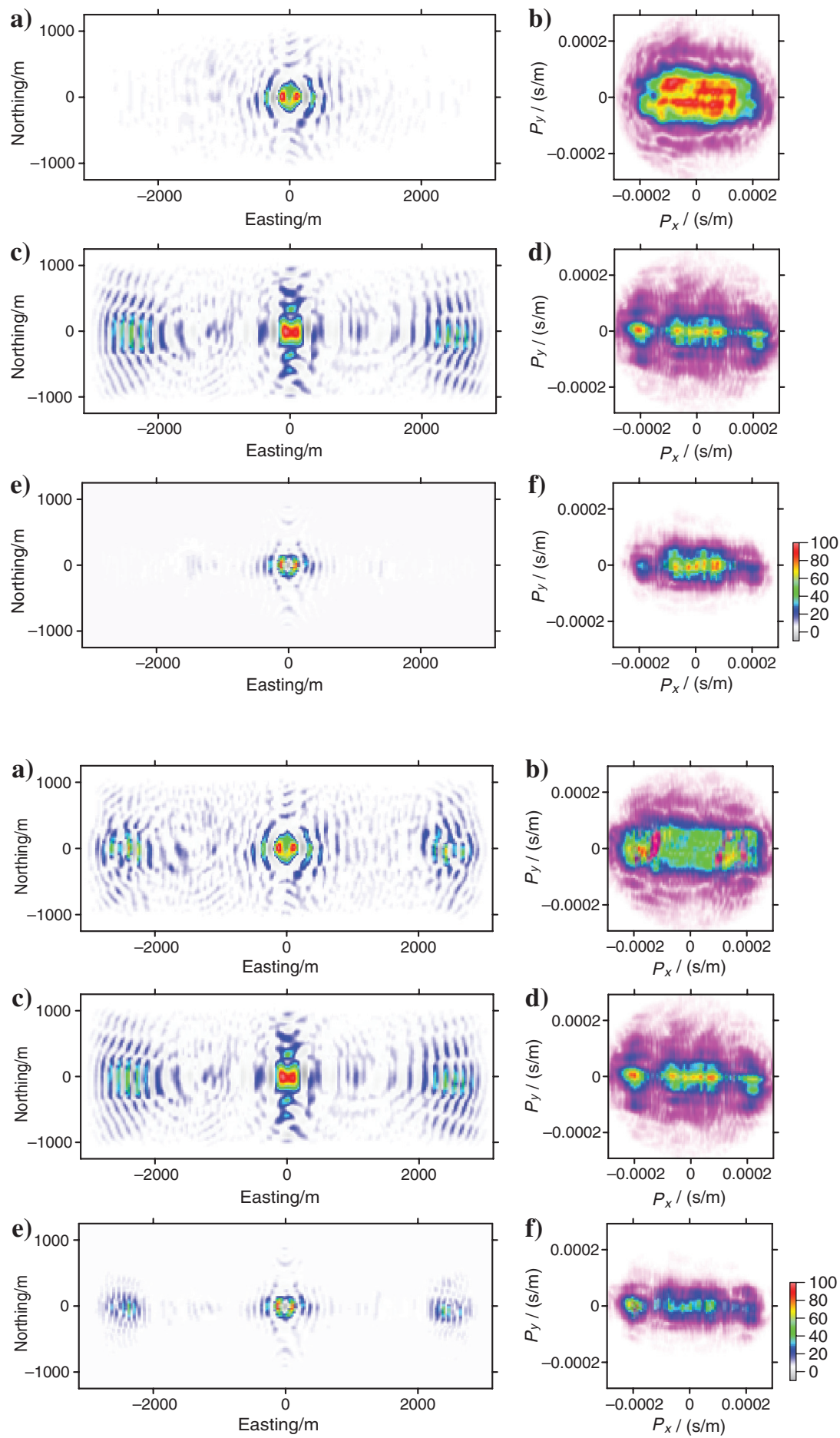

Figure 12. Multifrequency focal beams at depth $2313 \mathrm{~m}$ for acquisition Scheme I. (a) Detector beam, (b) Detector beam in the Radon domain, (c) Source beam, (d) Source beam in the Radon domain, (e) Spatial resolution function, and (f) AVP imprints. The colors indicate the amplitudes on a linear scale.
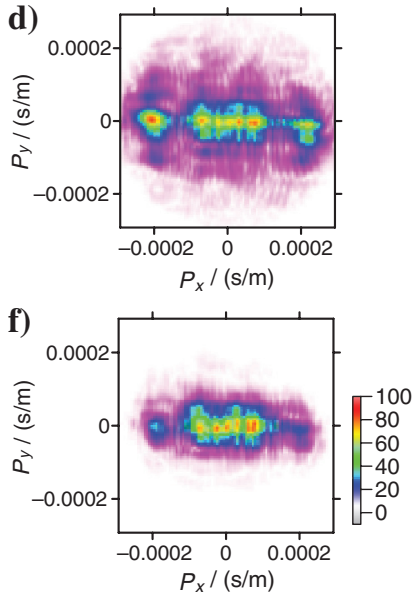

Figure 13. Multifrequency focal beams at depth $2313 \mathrm{~km}$ for acquisition Scheme II (a) Detector beam, (b) Detector beam in the Radon domain, (c) Source beam, (d) Source beam in the Radon domain, (e) Spatial resolution function, and (f) AVP imprints. The colors indicate the amplitudes on a linear scale. 
over the full seismic frequency bandwidth. We compute the multifrequency beams from only one single-frequency beam. Therefore, our method is considerably faster than the direct computation of multifrequency beams from all single-frequency beams involved, which makes multifrequency focal beam analysis feasible.

Note that the main factor affecting the computational efficiency of multifrequency focal beam analysis is the method chosen to simulate forward and inverse wave propagation, respectively. To achieve an acceptable trade-off between numerical accuracy and computation time for given computer resources, we introduced the DF-BK method for fast numerical wave propagation; this combines the 3D DF operator and the 3D BK wavefield interpolation.

The size of the extrapolation step for the two operators is important to ensure the accuracy of the extrapolation. Because the DF method only uses Fourier transforms for the propagation of wavefields, we can choose a large extrapolation step size to simulate the passage of a wave through a thick slab without concern for grid dispersion. Fu (2004) demonstrated that the ideal time-domain step size of the BK method for the very complex model like SEG/EAGE Salt Model is $32 \mathrm{~ms}$, which indicates that the ideal step size in the spatial domain is variable. The step of the DF-BK method greatly depends on the BK operator used in the wavefield interpolation in small layers, which have a higher step standard than the DF operator.

The velocity model in our method is a macrovelocity model. Therefore, an imprecise macrovelocity model may cause inexact results (Van Veldhuizen et al., 2008). If the uncertainty in the macrovelocity model is large, we should use a number of different scenarios to analyze the acquisition geometry.

\section{CONCLUSION}

In this paper, we propose a method for the efficient computation of 3D multifrequency focal beams, in which only one singlefrequency focal beam computation is required, followed by a number of interpolations. Conventional single-frequency focal beams can therefore be extended to a certain frequency range without greatly increasing computation costs.

The 3D wavefield extrapolation in our focal beam computation is conducted by the combined application of a 3D DF migrator and a 3D BK interpolation operator, which reduces computational costs for complex media.

We applied our method to a 3D model from an oil field in China. Because the multifrequency focal beams have a wider temporal frequency band than the single-frequency focal beams, their side-lobe energy distribution is more diffuse than that of the single-frequency focal beams. This is caused by destructive interference of the different frequency components involved. As expected, the main lobes in the focal beams are almost identical for schemes with different spatial sampling intervals, but with the same aperture, implying that they have a similar spatial resolution. However, the scheme for the smaller channel intervals shows a more uniform AVP imprint for the inline direction and it has smaller side lobes in the spatial resolution function. Therefore, the scheme with the smaller channel intervals is more favorable for seismic imaging and amplitude inversion.

\section{ACKNOWLEDGMENTS}

We are deeply grateful to Dr. Edith Miller for her constructive review to improve the paper and her help in editing the manuscript. The valuable comments by Dr. José M. Carcione and anonymous reviewers also are acknowledged. This research was supported by the Natural Science Foundation of China (Grant No. 41130418 and 40925013) and the National Basic Research Program (2009CB219403).

\section{APPENDIX A}

\section{BORN DISPERSION RELATIONSHIP FOR THE ONE-WAY APPROXIMATION SOLUTION}

We normalize the wavenumbers $\bar{k}_{x}=k_{x} / k_{0}, \bar{k}_{y}=k_{y} / k_{0}$, and $\bar{k}_{z}=k_{z} / k_{0}^{\prime}$, where $k_{0}^{\prime}$ is the background wavenumber of adjacent media.

For convenience, we take the refractive index $n(\mathbf{r})$ of the slab (Figure 3) as different constants $n$ to avoid the convolution operation in $F\left(k_{x}, k_{y}, z+\Delta z\right)$, implying that the following analysis within the Fourier domain proceeds by decomposing the heterogeneous slab into a series of heterogeneous slabs. In this sense, equation 13 can be written as

$$
\begin{aligned}
& u\left(k_{x}, k_{y}, z+\Delta z\right)\left[1-\frac{i k_{0} \Delta z O(n)}{2\left(\bar{k}_{z}+\bar{k}_{z}^{\prime}\right)}\right] \\
& =u\left(k_{x}, k_{y}, z\right)\left[1+\frac{i k_{0} \Delta z O(n)}{2\left(\bar{k}_{z}+\bar{k}_{z}^{\prime}\right)}\right] \exp \left(i k_{z} \Delta z\right),
\end{aligned}
$$

where $O(\mathbf{r})$ is the relative slowness perturbation defined as $O(\mathbf{r})=$ $n(\mathbf{r})-1$ with the refractive index $n(\mathbf{r})=v_{0} / v(\mathbf{r})$ and $i$ is the imaginary unit. Equation A-1 can be written as

$$
\begin{aligned}
& u\left(k_{x}, k_{y}, z+\Delta z\right) \\
& \quad=u\left(k_{x}, k_{y}, z\right) \exp \left[i 2 \arctan \frac{k_{0} \Delta z O(n)}{2\left(\bar{k}_{z}+\bar{k}_{z}^{\prime}\right)}\right] \exp \left(i k_{z} \Delta z\right) .
\end{aligned}
$$

Using the Born approximation, we obtain

$$
\arctan \frac{k_{0} \Delta z O(n)}{2\left(\bar{k}_{z}+\bar{k}_{z}^{\prime}\right)}=\frac{k_{0} \Delta z O(n)}{2\left(\bar{k}_{z}+\bar{k}_{z}^{\prime}\right)} .
$$

Substituting equation A-4 into equation A-3 gives

$$
\begin{aligned}
& u\left(k_{x}, k_{y}, z+\Delta z\right) \\
& \quad=u\left(k_{x}, k_{y}, z\right) \exp \left[\frac{i k_{0} \Delta z O(n)}{\left(\bar{k}_{z}+\bar{k}_{z}^{\prime}\right)}\right] \exp \left(i k_{z} \Delta z\right) .
\end{aligned}
$$

From equation A-5, we obtain the following Born dispersion relationship for the one-way approximation solution equation 14 to the heterogeneous slab.

$$
\bar{k}_{z}=\sqrt{1-\bar{k}_{x}^{2}-\bar{k}_{y}^{2}}+\frac{2(n-1)}{\sqrt{1-\bar{k}_{x}^{2}-\bar{k}_{y}^{2}}+\sqrt{1-\bar{k}_{x}^{\prime 2}-\bar{k}_{y}^{\prime 2}}}
$$


Let $\bar{k}_{x} \approx \bar{k}_{x}^{\prime}$ and $\bar{k}_{y} \approx \bar{k}_{y}^{\prime}$ without considering the refraction wave, and then substitute them in equation A-6 to yield the following dispersion equation

$$
\bar{k}_{z} \sqrt{1-\bar{k}_{x}^{2}-\bar{k}_{y}^{2}}+(n-1)\left(1-\bar{k}_{x}^{2}-\bar{k}_{y}^{2}\right)^{-1 / 2} .
$$

\section{REFERENCES}

Aminzadeh, F., J. Brac, and T. Kunz, 1997, 3-D Salt and Overthrust Models: SEG, SEG/EAGE 3-D Modeling Series No.1.

Bamberger, A., B. Engquist, L. Halpern, and P. Joly, 1988, Higher order paraxial wave equation approximations in heterogeneous media: SIAM Journal on Applied Mathematics, 48, 129-154, doi: 10.1137/ 0148006.

Berkhout, A. J., 1982, Seismic migration, imaging of acoustic energy by wavefield extrapolation, a theoretical aspects: Elsevier.

Berkhout, A. J., 1987, Applied seismic wave theory, advances in exploration geophysics: Elsevier.

Berkhout, A. J., 1997, Pushing the limits of seismic imaging, Part I: Prestack migration in terms of double dynamic focusing: Geophysics, 62 , 937-953, doi: 10.1190/1.1444201.

Berkhout, A. J., 2004, The data-driven seismic value chain, providing a business context for the velocity issue: Geophysical Prospecting, 52, 481-487, doi: 10.1111/gpr.2004.52.issue-6.

Berkhout, A. J., L. OngKiehong, A. W. F. Volker, and G. Blacquière, 2001, Comprehensive assessment of seismic acquisition geometry by focal beams - Part I: Theoretical considerations: Geophysics, 66, 911-917, doi: 10.1190/1.1444981.

Beylkin, G., 1985, Imaging of discontinuities in the inverse scattering problem by inversion of a causal generalized Radon transform: Journal of Mathematical Physics, 26, no. 1, 99-108, doi: 10.1063/1.526755.

Chang, T., C. Kue, L. Canales, and C. Shih, 2002, Forward modeling attribute analysis for AVO and prestack depth migration: 72nd Annual International Meeting, SEG, Expanded Abstract, 48-51.

Collins, M. D., 1993, A split-step Padé solution for the parabolic equation method: Journal of the Acoustical Society of America, 93, 1736-1742, doi: $10.1121 / 1.406739$.

Cordsen, A., M. Galbraith, and J. Peirce, 2000, Planning land 3-D seismic surveys: SEG

Fu, L. Y., 2004, Wavefield interpolation in the Fourier wavefield extrapolation: Geophysics, 69, 257-264, doi: 10.1190/1.1649393.

Fu, L. Y., 2006, Comparison of different one-way propagators for wave forward propagation in heterogeneous crustal wave guides: Bulletin of the Seismological Society of America, 96, no. 3, 1091-1113, doi: 10.1785/ 0120050159

Fu, L. Y., Y. G. Mu, and H. J. Yang, 1997, Forward problem of nonlinear Fredholm integral equation in reference medium via velocity- weighted wavefield function: Geophysics, 62, 650-656, doi: 10.1190/ 1.1444173 .

Gibson, R. L., and C. Tzimeas, 2002, Quantitative measures of image resolution for seismic survey design: Geophysics, 67, 1844-1852, doi: 10.1190/1.1527084.

Holberg, O., 1988, Towards optimum one-way wave propagation: Geophysical Prospecting, 36, 99-114, doi: 10.1111/gpr.1988.36.issue-2.

Huang, L. J., M. C. Fehler, and R. S. Wu, 1999, Extended local Born Fourier migration method: Geophysics, 64, 1524-1534, doi: 10.1190/ 1.1444656.

Muerdter, D., and D. Ratcliff, 2001, Understanding subsalt illumination through ray-trace modeling, Part 1: Simple 2-D salt models: The Leading Edge, 20, 578-595, doi: 10.1190/1.1438998.

Ristow, D., and T. Ruhl, 1994, Fourier finite-difference migration: Geophysics, 59, 1882-1893, doi: 10.1190/1.1443575.

Slawson, S. E., K. D. Grove, and G. W. Fischer, 1994, Model-based 3-D seismic acquisition design: 64th Annual International Meeting, SEG Expanded Abstracts, 919-920.

Stoffa, P. L., J. T. Fokkema, R. M. D. Freire, and W. P. Kessinger, 1990 Split-step fourier migration: Geophysics, 55, 410-421, doi: 10.1190/ 1.1442850 .

Thomson, D. J., 1990, Wide-angle parabolic equation solutions to two range-dependent benchmark problems: Journal of the Acoustical Society of America, 87, 1514-1520, doi: 10.1121/1.399450.

Trefethen, L. N., and L. Halpern, 1986, Well-posedness of one-way equations and absorbing boundary conditions: Mathematics of Computation, 47, 421-421, doi: 10.1090/S0025-5718-1986-0856695-2.

Tzimeas, C., 2004, Image resolution analysis: A new robust approach to seismic survey design: Ph.D. thesis, Texas A\&M University.

Van Veldhuizen, E. J., 2006, Integrated approach to 3-D seismic acquisition geometry analysis: Ph.D. thesis, Delft University of Technology.

Van Veldhuizen, E. J., G. Blacquière, and A. J. Berkhout, 2008, Acquisition geometry analysis in complex 3D media: Geophysics, 73, no. 5, Q43-Q58, doi: 10.1190/1.2972029.

Vermeer, G. J. O., 1999, Factors affecting spatial resolution: Geophysics, 64, 942-953, doi: 10.1190/1.1444602.

Vermeer, G. J. O., 2002, 3-D seismic survey design: SEG.

Volker, A. W. F., 2002, Assessment of 3-D seismic acquisition geometry by focal beams analysis: Ph.D. thesis, Delft University of Technology.

Volker, A. W. F., G. Blacquière, A. J. Berkhout, and L. OngKiehong, 2001 Comprehensive assessment of seismic acquisition geometry by focal beams - Part II: Practical aspects and examples: Geophysics, 66 918-931, doi: 10.1190/1.1444982.

Wu, R. S., and L. Chen, 2006, Directional illumination analysis using beamlet decomposition and propagation: Geophysics, 71, no. 4, S147-S159, doi: $10.1190 / 1.2204963$

Xie, X. B., S. Jin, and R. S. Wu, 2006, Wave-equation-based seismic illumination analysis: Geophysics, 71, no. 5, S169-S177, doi: 10.1190/ 1.2227619

Zhang, J. H., W. M. Wang, L. Y. Fu, and Z. X. Yao, 2008, 3D Fourier finitedifference migration by ADI plus interpolation: Geophysical Prospecting, 56, 95-103, doi: 10.1111/gpr.2008.56.issue-1. 